\title{
The quantum compass chain in a transverse magnetic field
}

\author{
Mostafa Motamedifar, Saeed Mahdavifar, Saber Farjami Shayesteh \\ Department of Physics, University of Guilan, \\ 41335-1914, Rasht, Iran
}

(Dated: November 9, 2018)

\begin{abstract}
We study the magnetic behaviors of a spin-1/2 quantum compass chain (QCC) in a transverse magnetic field, by means of the analytical spinless fermion approach and numerical Lanczos method. In the absence of the magnetic field, the phase diagram is divided into four gapped regions. To determine what happens by applying a transverse magnetic field, using the spinless fermion approach, critical fields are obtained as a function of exchanges. Our analytical results show, the field-induced effects depend on in which one of the four regions the system is. In two regions of the phase diagram, the Ising-type phase transition happens in a finite field. In another region, we have identified two quantum phase transitions in the ground state magnetic phase diagram. These quantum phase transitions belong to the universality class of the commensurate-incommensurate phase transition. We also present a detailed numerical analysis of the low energy spectrum and the ground state magnetic phase diagram. In particular, we show that the intermediate state $\left(h_{c_{1}}<h<h_{c_{2}}\right)$ is gapful, describing the spin-flop phase.

Email: m.motamedifa@guilan.ac.ir mahdavifar@guilan.ac.ir saber@guilan.ac.ir

PACS numbers: 75.10.Jm Quantized spin models;75.10.Pq Spin chain models
\end{abstract}

\section{INTRODUCTION}

Finding out the reason of different behaviors of quantum magnets has attracted much interest in recent years. It is known that the interaction between atoms plays a major role. Recently, theoretical works are focused on the important role played by the orbital degree of freedom in determining the magnetic properties of quantum magnets. The complex interplay among the spin and these types of degrees of freedom in quantum magnets, makes their phase diagram rich and induces various fascinating physical phenomena. Also, in some phenomena such as high-temperature superconductivity and colossal magnetic resistance, the orbital degree of freedom of electrons displays an important role.

The experimental observations on Mott insulators are a realization of the effect of the orbital degree of freedom on the low-energy behavior of a system. A proposed tactic to investigate these systems is based on the so-called quantum compass mode ${ }^{\underline{1}}$. In fact, the quantum compass model is a very good candidate for explaining the lowtemperature behavior of some Mott insulators. In this model, the orbital degrees of freedom are represented by (pseudo)spin-1/2 operators and coupled anisotropically in such a way to mimic the competition between orbital ordering in different directions.

The two-dimensional quantum compass model is introduced as a realistic model to generate protected cubits 22 and can play a role in the quantum information theory. This model is dual to studied model of superconducting arrays ${ }^{3}$. It was shown that the eigenstates are twofold degenerate and to be gapped $^{2}$. The results from both spin wave study and exact diagonalization have suggested a first-order quantum phase transition in the ground state phase diagram ${ }^{4}$. The existing of the firstorder phase transition is confirmed in two-dimensional quantum compass mode $\underline{1}^{5,6}$. Recently, it is found that in the ground state, most of the two-site spin correlations vanish and the two-dimer correlations exhibit the nontrivial hidden order ${ }^{7}$. Another examination of the $2 \mathrm{D}$ quantum compass model showed that a second order phase transition can occur when the frustration of exchange interaction increases ${ }^{8}$. In addition, finite temperature properties have been studied. It is found that the low-temperature ordered phase with a thermal transition corresponds to the 2D Ising universality class $\frac{9,10}{}$ and due to the dilution, the decrease of ordering temperature is much stronger than that in spin models 11 .

The $1 \mathrm{D}$ version is known as the quantum compass chain (QCC) and represents one particular subclass of low-dimensional quantum magnets which poses interesting theoretical problems ${ }^{12}-17$. By mapping the model to a quantum Ising chain, an exact solution is obtained for the ground state energy and the complete excitation spectrum 12 . It is shown that the QCC exhibits a firstorder phase transition at $J_{1}=0$ between two disordered phases with opposite signs of certain local spin correlations. The model is also diagonalized exactly by a direct Jordan-Wigner transformation 14 . The obtained results by latter approach, confirm the existence of the firstorder phase transition in the ground state phase diagram. In a very interesting work, it is found that the reported first-order phase transition, in fact occurs at a multicritical point where a line of the first-order transition meets with a line of the second-order transition 16 . Based on a numerical analysis 17 , the first and second order quan- 
tum phase transitions in the ground state phase diagram have been identified. By a detailed analysis of the numerical results on the spin structure factors, it is shown that the Néel and the stripe-antiferromagnet long-range orders exist in the ground state phase diagram 17 .

The effect of a transverse magnetic field on the QCC is studied by Ke-Wei Sun et al.18. They allowed the changing a parameter that only provides moving on the first order critical line. Opening the energy gap of the system when a magnetic field applied is the result that they could achieve. In spite of the calculation of pseudo-spin correlation functions, fidelity susceptibility, the concurrence, the block-block entanglement entropy, they didn't investigate the ground state phase diagram of the QCC in a transverse magnetic field. They only restricted their study on the first order critical line. In a very recent work $^{19}$, our group studied the presence QCC in an area of the ground state magnetic phase diagram where the odd couplings are antiferromagnetic and larger than even couplings. Using pseudo spin ladder operators indicated that the QCC in a transverse magnetic field can be mapped to the one dimensional Ising model in a transverse magnetic field (ITF). Analytical investigation of the effective ITF Hamiltonian predicts the occurrence of two quantum phase transitions by increasing magnetic field.

In this paper we use the main idea of Perk ${ }^{21}$ to provide analytical results on the effect of a transverse magnetic field in whole range of the ground state phase diagram. Depending on values of the couplings, one or two quantum phase transitions can happen in finite critical fields. To make a clear picture of different long-range order phases, we do a very accurate numerical experiment. In particular we use the numerical Lanczos method to diagonalize numerically finite chains up to $N=24$ spins. Based on the exact diagonalization results we show that the spin-flop long-range order induces between two critical magnetic fields. The spin-flop phase is denoted as a phase with the staggered magnetization perpendicular to the direction of the transverse magnetic field.

The outline of the paper is as follows. In forthcoming section we introduce the results of the analytical fermionization studies. In the section III we discuss the model in the strong exchange couplings limit and derive the effective spin chain Hamiltonian. In section IV, we present results of an accurate numerical experiment. Finally, we have discussed and summarized our results in section $\mathrm{V}$.

\section{FERMIONIZATION}

The Hamiltonian of the QCC in a transverse magnetic field with $N$ periodic sites is given by 16,17

$$
\begin{aligned}
\mathcal{H} & =\sum_{i=1}^{N / 2} J_{1} \sigma_{2 i-1}^{z} \sigma_{2 i}^{z}+J_{2} \sigma_{2 i-1}^{x} \sigma_{2 i}^{x}+L \sigma_{2 i}^{z} \sigma_{2 i+1}^{z} \\
& -h \sum_{i=1}^{N} \sigma_{i}^{y} .
\end{aligned}
$$

Here $\sigma_{i}^{x, z}$ are the Pauli operators on the $i$ th site and $J_{1}, J_{2}, L_{1}$ are the exchange couplings. $h$ denotes the transverse magnetic field. In the absence of the transverse magnetic field, $h=0$, the first and second order critical lines denote with $J_{1} / L_{1}=0$ and $J_{2} / L_{1}=1$, respectively ${ }^{16}$. There are four gapped phases in the regions: (I.) $J_{1} / L_{1}>0, J_{2} / L_{1}>1$, (II.) $J_{1} / L_{1}>0$, $J_{2} / L_{1}<1$, (III.) $J_{1} / L_{1}<0, J_{2} / L_{1}<1$, (IV.) $J_{1} / L_{1}<0$, $J_{2} / L_{1}>1$. There is a hidden order in the regions (I.) and (IV.). But two kind of magnetic long-range orders, a type of the Néel and the stripe-antiferromagnetic orders have been recognized in the regions (II.) and (III.) respectively 17 .

A mapping of the spin- $\frac{1}{2}$ operators onto fermi operators by means of the jordan-Wigner transformation was vastly used by many physicists who are interested to investigate the Hamiltonian of the system by the aid of the spinless fermions. Because there are alternating links in the QCC we introduce two kinds of spinless fermion through the following Jordan-Wigner transitions ${ }^{22}$ :

$$
\begin{aligned}
\mathcal{S}_{2 n-1}^{+} & =a^{\dagger} e^{i \pi \sum_{m=1}^{n-1}\left(a_{m}^{\dagger} a_{m}+b_{m}^{\dagger} b_{m}\right)} \\
\mathcal{S}_{2 n}^{+} & =b^{\dagger} e^{i \pi\left(\sum_{m=1}^{n} a_{m}^{\dagger} a_{m}+\sum_{m=1}^{n-1} b_{m}^{\dagger} b_{m}\right)} \\
\mathcal{S}_{2 n-1}^{y} & =a_{n}^{\dagger} a_{n}-\frac{1}{2}, \mathcal{S}_{2 n}^{y}=b_{n}^{\dagger} b_{n}-\frac{1}{2} .
\end{aligned}
$$

Using the above transformation, the QCC is mapped to a 1D interacting spinless fermion system:

$$
\begin{aligned}
\mathcal{H}_{f} & =\left(\frac{J_{1}-J_{2}}{4}\right) \sum_{n=1}^{\frac{N}{2}}\left[a_{n}^{\dagger} b_{n}^{\dagger}-a_{n} b_{n}\right] \\
& +\left(\frac{J_{1}+J_{2}}{4}\right) \sum_{n=1}^{\frac{N}{2}}\left[a_{n}^{\dagger} b_{n}-a_{n} b_{n}^{\dagger}\right] \\
& +\frac{L}{4} \sum_{n=1}^{\frac{N}{2}}\left[b_{n}^{\dagger} a_{n+1}^{\dagger}-b_{n} a_{n+1}-b_{n} a_{n+1}^{\dagger}+b_{n}^{\dagger} a_{n+1}\right] \\
& -h \sum_{n=1}^{\frac{N}{2}}\left[\left(a_{n}^{\dagger} a_{n}-\frac{1}{2}\right)+\left(b_{n}^{\dagger} b_{n}-\frac{1}{2}\right)\right] .
\end{aligned}
$$

Then by means of Fourier transformations, the mean field Hamiltonian is given by

$$
\begin{aligned}
\mathcal{H}_{M F} & =\left(\frac{J_{1}-J_{2}}{4}\right) \sum_{q}\left[A_{q}^{\dagger} B_{-q}^{\dagger}+B_{-q} A_{q}\right] \\
& +\sum_{q}\left(\frac{J_{1}+J_{2}+L e^{-i q}}{4}\right)\left[A_{q}^{\dagger} B_{q}\right] \\
& +\sum_{q}\left(\frac{J_{1}+J_{2}+L e^{+i q}}{4}\right)\left[B_{q}^{\dagger} A_{q}\right] \\
& +\left(\frac{L}{4}\right) \sum_{q}\left[e^{+i q} B_{q}^{\dagger} A_{-q}^{\dagger}+e^{-i q} A_{-q} B_{q}\right] \\
& -h \sum\left(A_{q}^{\dagger} A_{q}+B_{q}^{\dagger} B_{q}\right) .
\end{aligned}
$$


Finally, by diagonalizing the mean field Hamiltonian 21 , the critical transverse fields obtain as

$$
\begin{aligned}
& h_{c_{1}}=\sqrt{J_{1}\left(L+J_{2}\right)} \\
& h_{c_{2}}=\sqrt{-J_{1}\left(L-J_{2}\right)} .
\end{aligned}
$$

Surprizing is that, by applying the transverse magnetic field two quantum phase transitions can occur in the region (I.). But in the regions (II.) and (III.), there is only one critical field. In the region (IV.), no quantum phase transition occurs by increasing the transverse magnetic field in a finite value of $h$.

\section{EFFECTIVE HAMILTONIAN}

In this section, we briefly discuss the model (11) in limiting case of the exchanges $\frac{J_{1}}{L}=\frac{J_{2}}{L}=\frac{J}{L} \gg 0$. In this limit the model (11) can be mapped onto the effective spin chain Hamiltonian $\underline{19}$.

In order to work easily, we rotated the system in the special sort that the field oriented in $z$ direction. In the absence of the magnetic field, we consider one of the odd links for example the first link. For this dimer the quantum state is either the singlet state, $|S\rangle$, with the energy eigenvalue $-J / 2$ or triplet state, $\left|T_{1}\right\rangle,\left|T_{-1}\right\rangle,\left|T_{0}\right\rangle$ with energy eigenvalues of $0,0, J / 2$ respectively.

At $h=0$ the ground state of a distinct dimer is $|S\rangle$. As the magnetic field $h$ increases the energy of the triplet state $\left|T_{1}\right\rangle$ decreases and at $h=J / 2$ forms together with the singlet state, a doublet of almost degenerate low energy state. This degenerate state splits from the remaining high energy two triplet states. Thus, for a strong enough magnetic field we have a situation when the singlet $|S\rangle$ and triplet $\left|T_{1}\right\rangle$ states create a new effective spin $\tau=1 / 2$ system. On the new singlet-triplet subspace and up to a constant, one can easily obtain the effective Hamiltonian 19

$$
\mathcal{H}^{e f f}=\frac{L}{2} \sum_{n=1} \tau_{n}^{x} \tau_{n+1}^{x}-(h-J) \sum_{n=1} \tau_{n}^{z} .
$$

The effective Hamiltonian describes the Ising chain in an effective transverse magnetic field ${ }^{20} h^{\text {eff }}=h-J$. The critical effective fields for the Ising chain in a transverse magnetic field (ITF) are rigorously interesting, one of them is $h_{c_{1}}^{\text {eff }}=-L / 2$ and the other one is $h_{c_{2}}^{\text {eff }}=L / 2$. Therefore critical fields of the main QCC can be obtained as

$$
\begin{aligned}
& h_{c_{1}}=J-L / 2 \\
& h_{c_{2}}=J+L / 2 .
\end{aligned}
$$

It is clear that the hidden ordered gapped phase at $h^{e f f}<h_{c_{1}}^{e f f}$ for the QCC corresponds to the negatively saturated magnetization phase for the effective ITF model. Whereas the intermediate gapped phase at $h_{c_{1}}^{e f f}<h^{e f f}<h_{c_{2}}^{e f f}$ for the QCC corresponds to the Néel phase for the effective ITF model. Finally, the region $h^{\text {eff }}>h_{c_{2}}^{e f f}$ corresponds to the fully magnetization phase of the effective ITF model where the QCC is totally magnetized. In the next section, we present our numerical results obtained by a very accurate numerical experiment and confirm the mentioned quantum phase transitions.

\section{NUMERICAL EXPERIMENT}

By doing an experiment, the validity of the suggested theoretical results on the effect of a transverse magnetic field on the QCC, can be determined. Since a real experiment cannot be done at zero temperature, the best way is doing a virtual numerical experiment. A very famous and accurate method in field of the numerical experiments is known as the Lanczos method. The Lanczos method and the related recursion methods ${ }^{23,24}$, possibly with appropriate implementations, have emerged as one of the most important computational procedures, mainly when a few extreme eigenvalues are desired. However, the strong role of a numerical experiment to examine quantum phase transitions is not negligible.

In this section, to explore the nature of the spectrum and the quantum phase transition, we used Lanczos method to diagonalize numerically chains with length up to $N=24$ and different values of the exchanges. The energies of the few lowest eigenstates were obtained for chains with periodic boundary conditions.

The first information that can be gained is the energy gap. In this way we have accessed to the energy gap which is recognized as the difference between the second exited state and the ground state energy in finite QCC. In Fig 1 the energy gap is plotted versus transverse magnetic field, $h$, for various length of chains. This figure contains four graphs of the energy gap in different sectors of the ground state phase diagram. The Fig.1(a) devoted to the energy gap of the system at the area (I.) of the phase diagram. In this figure the computed energy gap corresponding various length of chains $(N=12,16,20)$ and exchanges $L=1.0, J_{1}=3.0$ and $J_{2}=3.0$ is plotted versus the transverse magnetic field. By increasing the size of the system the energy gap curve will approximate to the magnetic field axes. The magnetic fields in which the energy gap in the thermodynamic limit will be closed, are critical fields. By means of the phenomenological renormalization group technique $\underline{26}$ we have found $h_{c 1}=2.5 \pm 0.1$ and $h_{c 2}=3.5 \pm 0.1$ for critical fields in that the system undergos the quantum phase transition. In other words, in absence of the transverse magnetic field, the system is in a gapfull phase. By increasing the magnetic field, the gap of energy decreases and finally 

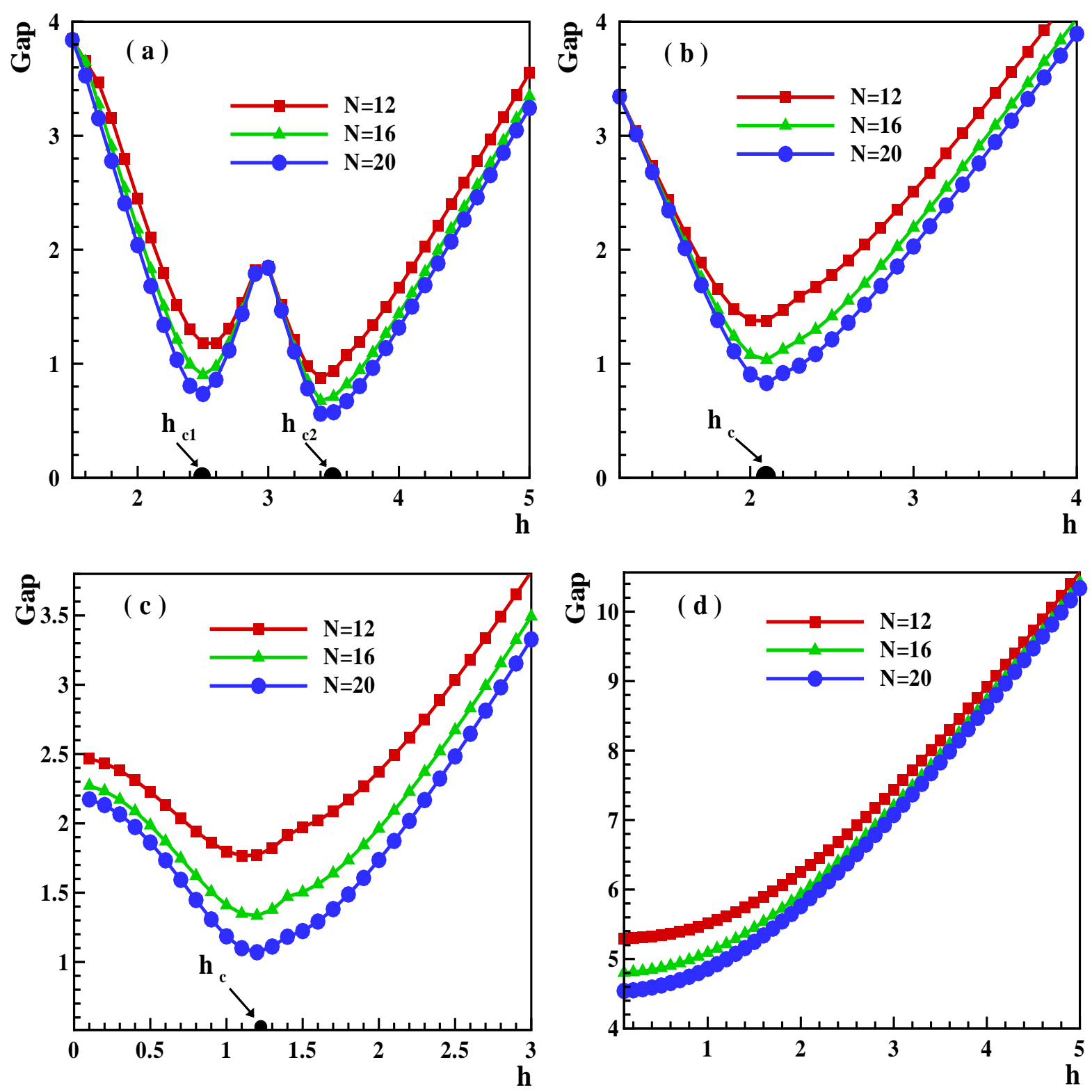

FIG. 1: (Color online.) The energy gap as a function of the transverse magnetic filed $h$, for different chain lengths $N=12,16,20$ and exchanges $L_{1}=1.0$, (a) $J_{1}=J_{2}=3.0$, (b) $J_{1}=3.0, J_{2}=0.5$, (c) $J_{1}=-3.0, J_{2}=0.5$ and (d) $J_{1}=-3.0, J_{2}=3.0$.

will close at the first critical field $h_{c 1}=2.5 \pm 0.1$. After first critical field the gap of energy will be opened by growing magnetic field, while at the second critical field $h=3.5 \pm 0.1$, again will be closed. For values of the magnetic field further than $h=3.5 \pm 0.1$ the gap of system will open and the system will go to a saturated phase. In conclusion, applying the transverse magnetic field to the gapped QCC in region (I.), creates two new gapfull magnetic phases. The numerical critical fields with selected amount of exchanges $L, J_{1}$ and $J_{2}$ precisely correspond with the previous analytical critical fields (Eq 5 and Eq77).

In figures 1(b) and 1(c), the numerical results on the energy gap are plotted for various length of chains,
$N=12,16,20$ and exchanges $L=1.0, J_{1}=3.0, J_{2}=0.5$, in region (II.) and $J_{1}=-3.0, J_{2}=0.5$, in region (III.). As it can be seen in Fig प(b) and (c), the qualitative effect of the transverse magnetic field in the regions (II.) and (III.) is equivalent. In the absence of the magnetic field, the system is gapped. Increasing the transverse magnetic filed, the energy gap decreases and will be closed in $h_{c}=2.1 \pm 0.1\left(h_{c}=1.2 \pm 0.1\right)$ in regions (II.) and (III.) respectively. More increasing the magnetic field from $h_{c}$, the energy gap opens again and behaves almost linearly. The obtained numerical critical fields in these regions are in well agreement with the analytical results (Eq 5). Finally, Fig [(d) allocated to the energy gap at the area (IV.) of the ground state phase diagram for chain 

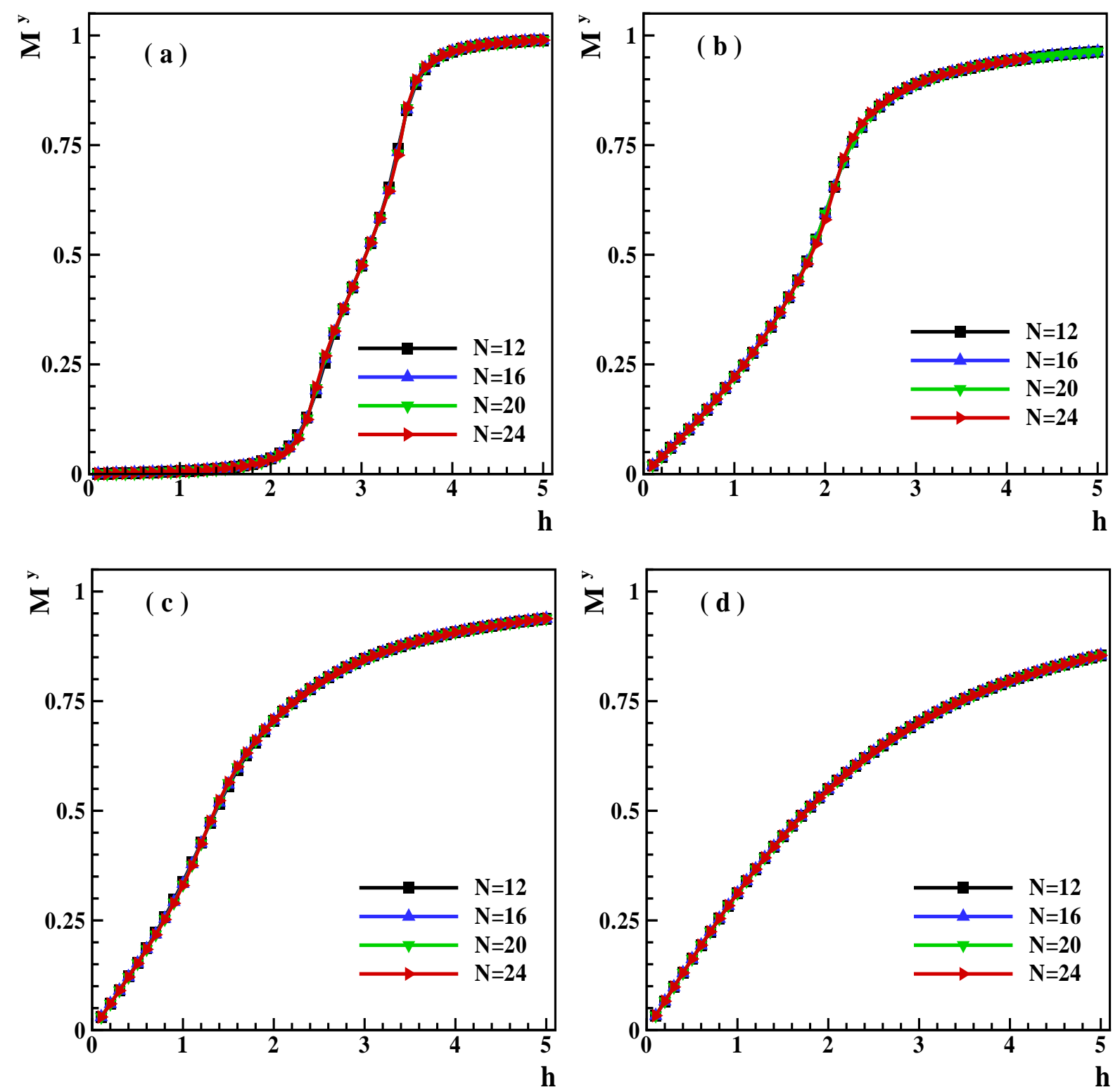

FIG. 2: (Color online.) The magnetization along the field $M^{y}$, as a function of the transverse magnetic filed $h$, for different chain lengths $N=12,16,20,24$ and exchanges $L_{1}=1.0$, (a) $J_{1}=J_{2}=3.0$, (b) $J_{1}=3.0, J_{2}=0.5$, (c) $J_{1}=-3.0, J_{2}=0.5$ and (d) $J_{1}=-3.0, J_{2}=3.0$.

sizes $N=12,16,20$, and exchanges $L=1.0, J_{1}=-3.0$, $J_{2}=3.0$. In this figure, is clearly seen that by increasing the transverse magnetic field, the energy spectrum of the QCC will remain gapfull. There aren't any critical magnetic fields. The system goes to a saturated magnetic phase for enough large values of the transverse magnetic field.

So far the paper was determined that the transverse field, depending on the values of the exchanges can induces two or one gapped phases in the ground state phase diagram of the QCC. Subsequently, a very important question that precedes is: "What are the magnetic long-range ordered phases in these gapped regions of the QCC?" A very clear answer can provides, doing the numerical Lanczos experiment. To recognize the different magnetic phases induced by transverse field, $h$, we implemented the algorithm for finite-size chains $(N=12,16,20,24)$ to calculate the order parameters and the various spin correlation functions.

An insight into the nature of different phases can be obtained by studying the magnetization process. The magnetization along the transverse field axis is defined as

$$
M^{y}=\frac{1}{N} \sum_{i=1}^{N}\left\langle G s\left|\sigma_{i}^{y}\right| G s\right\rangle
$$


where the notation $\langle G s|\ldots| G s\rangle$ represents the ground state expectation value. In Fig 2] we have plotted the transverse magnetization, $M^{y}$, versus $h$, for different chain sizes $N=12,16,20,24$ and different values of the exchanges that covered all regions in the ground state phase diagram. We did not find any size effect on the numerical results of the transverse magnetization. As is seen in Fig2(a), due to the profound effect of quantum fluctuations, the transverse magnetization remains small but finite for $0<h<h_{c_{1}}$ and reaches zero at $h=0$. This behavior shows that in the gapped phase with hidden order in region (I.), the transverse magnetization appears only at a finite critical value of the transverse field, $h_{c_{1}}$. This phenomenon is also reported for a two-leg ladder ${ }^{25}$ and an alternating spin chain $\underline{26}$. For $h>h_{c_{1}}$, transverse magnetization increases almost linearly with increasing transverse field. At the second critical field $h_{c_{2}}$, due to the quantum fluctuations the magnetization do not saturate and reaching saturation asymptotically in the limit of infinite transverse field.

Numerical results on the magnetization function presented in figures 2(b) and 2(c) are computed for various length of chains $(N=12,16,20,24)$ and exchanges $L=1.0, J_{1}=3.0, J_{2}=0.5$, in region (II.) and $J_{1}=-3.0$, $J_{2}=0.5$, in region (III.). As the same as the energy gap function, the qualitative effect of the transverse magnetic field in the regions (II.) and (III.) is equivalent. In the absence of the transverse field, the transverse magnetization is zero. As soon as the transverse field is applied, the transverse magnetization start to increase. In the region (III.), the transverse magnetization increases faster than the region(II.). It is also completely clear that the system at the critical field $h_{c}$ do not saturate. The saturation will be happened for very larger values of the transverse field. Fig 2(d) shows the numerical results in the region (IV.) of the ground state phase diagram for chain sizes $N=12,16,20,24$, and exchanges $L=1.0$, $J_{1}=-3.0, J_{2}=3.0$. As soon as the transverse field is applied, the transverse magnetization starts to increase from zero. The transverse magnetization grows smoothly by increasing the transverse field and due to profound effect of the quantum fluctuations, it will be saturated in infinite transverse field. By comparing figures 2(a) and 2(d), one can find that the hidden order in the region (I.) must be completely different from the hidden order phase in the region (IV.). The hidden order phase in the region (I.), will not surrender versus the transverse field, but the hidden order phase in the region (IV.) as soon as the transverse field applied will surrender versus field and spins start to aline the field direction.

To find the long-range magnetic order of the ground state of the system, we start our consideration with the spin-spin correlation function defined by

$$
W_{j}^{\alpha \alpha}(n)=\left\langle\sigma_{j}^{\alpha} \sigma_{j+n}^{\alpha}\right\rangle \quad(\alpha=x, y, z),
$$

and the spin structure factor at momentum $q$ defined by

$$
S^{\alpha \alpha}(q)=\sum_{n=1}^{N-1} W_{j}^{\alpha \alpha}(n) \exp (i q n) .
$$

It is known that the spin structure factor give us a deep insight into the characteristics of the ground state $\mathrm{e}^{17}$.

By examining the energy gap we found that a new magnetic phase is induced by applying the transverse field in the region (I.) between $h_{c_{1}}<h<h_{c_{2}}$. To determine how to long-range order there is, in Fig 3 (a) we have plotted $W_{1}^{z z}$ as a function of $n$ for different values of the transverse field. The selected values of the transverse field $h=0.7,3.0,4.2$ in the figure cover all phases in the region (I.). It can be seen from this figure (Fig] (a)) that the $z$ component of the spins on odd sites is pointed in the same direction with the $\sigma_{1}^{z}$ and others (on even sites) are pointed in opposite direction for a value of $h=3.0$. This is an indication for the Néel ordering in the intermediate region $h_{c_{1}}<h<h_{c_{2}}$. This is very interesting that the numerical results suggest that a different novel phase can be induced by increasing the transverse field which is known as the spin-flop phase. For any value of the transverse field the Lanczos results lead to the staggered magnetization, $M_{s t}^{z}=\frac{1}{N} \sum_{j=1}^{N}(-1)^{j} \sigma_{j}^{z}=0$, since the ground state is degenerate and in a finite system no symmetry breaking happens. But, the $h$-dependency of the spin structure factor, $S^{z z}(\pi)$, is qualitatively the same as the staggered magnetization, $M_{s t}^{z}$. However the spinspin correlation function diverges in the ordered phase as $N \longrightarrow \infty$. We computed the spin structure factor $S^{z z}(\pi)$ and in Fig 3 (b), we have plotted $S^{z z}(q=\pi) /(N-1)$ as a function of $h$ for the chain lengths $N=12,16,20,24$ and exchanges $L=1.0, J_{1}=3.0, J_{2}=3.0$. It can be seen that in the intermediate region $h_{c_{1}}<h<h_{c_{2}}$, the ground state of the system is in the Néel phase. There is not the Néel ordering along $z$ axis in regions $h<h_{c_{1}}=2.5 \pm 0.1$ and $h>h_{c_{2}}=3.5 \pm 0.1$. To check the existence of the Néel order in the thermodynamic limit $N \longrightarrow \infty$ of the system in the intermediate region $h_{c_{1}}<h<h_{c_{2}}$, we have plotted in Fig B(c) the $N$ dependence of $S^{z z}(\pi)$ for different values of transverse field. Increasing behavior of the spin structure factor in the sector $h_{c_{1}}<h<h_{c_{2}}$, shows that the spin-flop phase is a true long-range order.

On the other hand the behavior of the energy gap versus the magnetic field in the regions (II.) and (III.), suggested a quantum phase transition at the critical field $h_{c}$. In a very recent work ${ }^{17}$, Néel and stripeantiferromagnetic long-range orders, in the absence of the field, have been reported in the regions (II.) and (III.) respectively. The "stripe-antiferromagnetic phase" is define as a phase with the opposite magnetization along the $z$ axis on the odd bonds. The order parameter of the stripe-antiferromagnetic phase is defined as $\frac{17}{}$

$$
M_{s p}^{z}=\frac{2}{N}\left\langle\sum_{j=1}^{N / 2}(-1)^{j}\left(\sigma_{2 j-1}^{z}+\sigma_{2 j}^{z}\right)\right\rangle .
$$



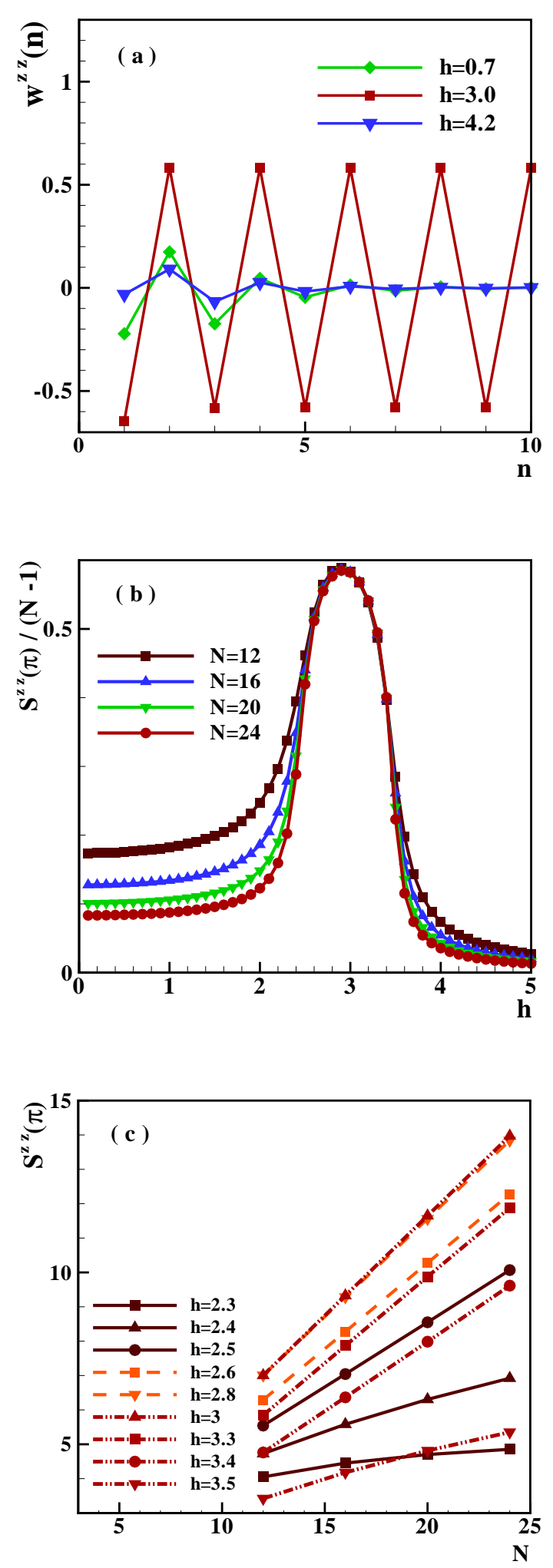

FIG. 3: (a) The spin-spin correlation $W_{1}^{z z}$ as a function of $n$, for chain length $N=20$ and exchanges $L_{1}=1.0, J_{1}=$ $J_{2}=3.0$. (b) The spin structure factor $S^{z z}(\pi) /(N-1)$ as a function of the transverse field $h$ for different chain lengths $N=12,16,20,24$ and exchanges $L_{1}=1.0, J_{1}=J_{2}=3.0$. (c) The spin structure factor $S^{z z}(\pi)$ plotted as a function of the chain length $N$, for different values of the field in the intermediate region.

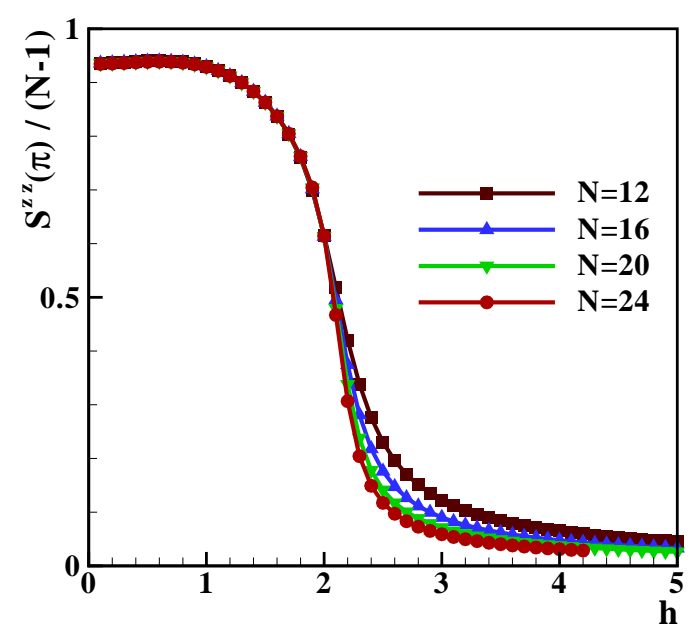

FIG. 4: (Color online.) The spin structure factor $S^{z z}(\pi) /(N-$ $1)$ as a function of the transverse field $h$ for different chain lengths $N=12,16,20,24$ and exchanges $L_{1}=1.0, J_{1}=$ $3.0, J_{2}=0.5$.

We computed the spin structure factor $S^{z z}(\pi)$ and the correlation function of the stripe-antiferromagnetic order parameter given by

$\chi^{z z}=\left\langle\sum_{n=1}^{N / 2-1}(-1)^{n}\left(\sigma_{2 j-1}^{z}+\sigma_{2 j}^{z}\right)\left(\sigma_{2 j-1+2 n}^{z}+\sigma_{2 j+2 n}^{z}\right)\right\rangle$.

In Fig 4 we have plotted $S^{z z}(\pi) /(N-1)$ as a function of $h$ for the chain lengths $N=12,16,20,24$ and exchanges $L=1.0, J_{1}=3.0, J_{2}=0.5$. Clearly be seen that the Néel order remains in the presence of the field up to the critical field $h_{c}=2.1 \pm 0.1$. Overlapping of the numerical results in the region $h<h_{c}=2.0 \pm 0.1$ shows a divergent behavior of the function $S^{z z}(\pi)$ by increasing the size of chain $N$. This justifies that the Néel ordering along the $z$ axis is true long-range order in the region $h<h_{c}=2.1 \pm 0.1$ of the ground state phase diagram. Induced quantum fluctuations by increasing $h$ from zero, decreases the staggered magnetization from the almost saturation value. We checked our numerical results and found that there is not the Néel ordering along $z$ axis in the region $h>h_{c}$.

The final insight into the nature of different phases be related to the effect of the field in the region (III.) Which can be obtained by studying the correlation func-

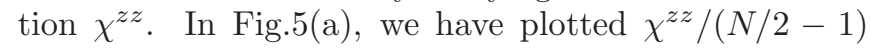
as a function of the field $h$ for different values of the chain length $N=12,16,20,24$ and exchanges $L=1.0$, $J_{1}=3.0, J_{2}=-0.5$. It is completely clear, that there is stripe-antiferromagnetic ordering along the $z$ axis in the region $h<h_{c}=1.2 \pm 0.1$. The induced quantum fluctuations by increasing $h$ from zero, de- 

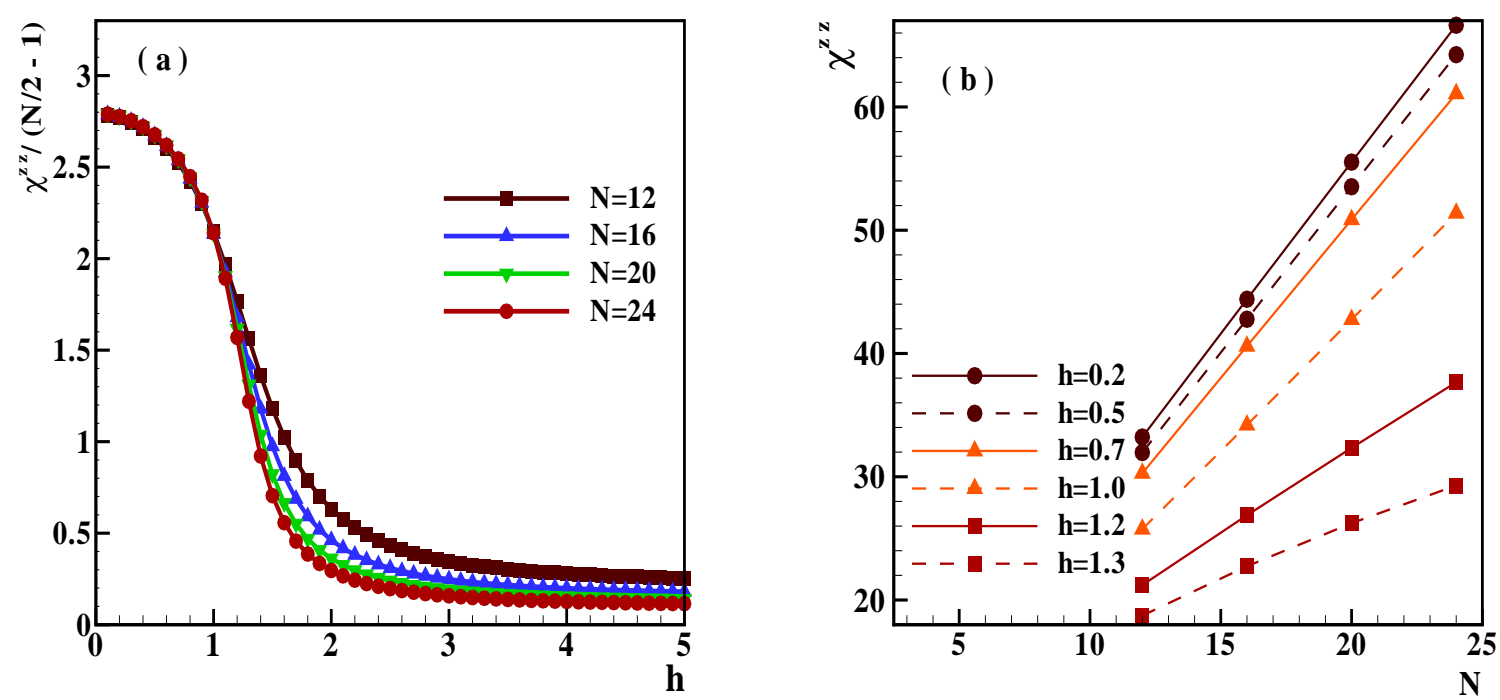

FIG. 5: (Color online.) (a) The correlation function of the stripe antiferromagnetic order parameter $\chi^{z z} /(N / 2-1)$ as a function of the transverse field $h$ for different chain lengths $N=12,16,20,24$ and exchanges $L_{1}=1.0, J_{1}=-3.0, J_{2}=0.5$. (b) The correlation function $\chi^{z z}$ versus the chain length $N$ for different values of the field and exchanges $L_{1}=1.0, J_{1}=-3.0, J_{2}=0.5$.

creases the stripe-antiferromagnetic order from almost saturation value. Again, to check the existence of the stripe-antiferromagnetic order in the thermodynamic limit $N \longrightarrow \infty$ of the system in the region $h<h_{c}=$ $1.2 \pm 0.1$, we have plotted in Fig 5 (b) the $N$ dependence of $\chi^{z z}$ for different values of field $h<h_{c}=1.2 \pm 0.1$. As is seen from this figure in the region $h<h_{c}=1.2 \pm 0.1$ there is a divergent behavior which shows that the stripeantiferromagnetic order is true long-range order. By investigating the $N$ dependence of $\chi^{z z}$ for different values of $J_{1} / L_{1}$, we found that there is no long-range stripe-antiferromagnetic order for fields larger than the $h_{c}=1.2 \pm 0.1$.

\section{CONCLUSION}

In this paper we considered the $1 \mathrm{D}$ quantum compass model (QCC) and built a clear picture of different magnetic phases induced by an external transverse magnetic field at zero temperature. This picture is made step by step. At the first step, using the analytical spinless fermion approach we found that, depending on the values of the couplings, by increasing the transverse magnetic field, one or two quantum phase transitions occur in the ground state magnetic phase diagram of the QCC. These quantum phase transitions belong to the universality class of the commensurate-incommensurate phase transition.

In the second step, we did an accurate numerical experiment using the Lanczos method. This part of our work, helped us to increasing the brightness of the ground state phase diagram picture. We have implemented the Lanczos method to numerically diagonalize finite chains. Using the exact diagonalization results, first we have calculated the energy gap. In complete agreement with our analytical results, we showed that depending on the values of the couplings, by increasing the transverse magnetic field, the energy gap closed at one or two critical fields. Then we also studied the magnetization process in all regions of the phase diagram. By comparing the magnetization curves, we showed that the suggested zero-field hidden ordered phases have different behaviors in presence of a transverse field. The hidden order phase in the region (I.), will not surrender versus the TF, but the hidden order phase in the region (IV.) as soon as the TF applied will surrender versus field and spins start to aline in the field direction. Finally, to distinguish the kind of magnetic orders we plotted the spin-spin correlation functions and found that the spin-flop long-range order can be induced in a sector of the ground state magnetic phase diagram.

\section{ACKNOWLEDGMENTS}

Authors would like to thank, J. H. H. Perk, H. Johannesson, E. Eriksson, and J. Abouie for useful comments and interesting discussions. MM would like to thank from A. Davody and H. R. Afshar for comments on mathematical calculations. 
${ }^{1}$ K. I. Kugel and D. I. Khomskii, Sov. Phys. Jetp 37, 725 (1973).

2 B. Doucot, M. V. Feigelman, L. B. Ioffe, and A. S. Ioselevich, Phys. Rev. B 71, 024505 (2005).

3 Z. Nussinov and E. Fradkin, Phys. Rev. B 71, 195120 (2005).

4 J. Dorier, F. Becca, and F. Mila, Phys. Rev. B 72, 024448 (2005).

${ }^{5}$ H. -D. Chen, C. Fang, J. Hu, and H. Yao, Phys. Rev. B 75, 144401 (2007).

6 R. Orus, A. C. Doherty, and G. Vidal, Phys. Rev. Lett. 102, 077203 (2009).

7 W. Brzezicki, and A. M. Oles, Phys. Rev. B(R) 82, 060401 (2010).

8 L. Cincio, J. Dziarmaga, A. M. Oles, Phys. Rev. B 82, 104416 (2010).

9 S. Wenzel and W. Janke, Phys. Rev. B 78, 064402 (2008); Phys. Rev. B 78, 099902(E) (2008).

10 A. Mishra, M. Ma, F. -C. Zhang, S. Guertler, L. -H. Tang, S. Wan, Phys. Rev. Lett. 93, 207201 (2004); Phys. Rev. B 78, 099902(E) (2008).

11 T. Tanaka and S. Ishihara, Phys. Rev. Lett. 98, 256402 (2007).

12 W. Brzezicki, J. Dziarmaga, and A. M. Oles, Phys. Rev. B 75, 134415 (2007).
13 W. -L. You and G. -S. Tian, Phys. Rev. B 78, 184406 (2008).

14 W. Brzezicki, and A. M. Oles, Acta Phys. Pol. A 115, 162 (2009).

15 Ke-Wei Sun, Yu-Yu Zhang, and Qing-Hu Chen, Phys. Rev. B 79, 104429 (2009).

16 Erik Eriksson, and Henrik Johannesson, Phys. Rev. B 79, 224424 (2009).

17 S. Mahdavifar, Eur. Phy. J. B 77, 77-82 (2010).

18 Ke-Wei Sun and Qing-Hu Chen, Phys. Rev. B 80, 174417 (2009).

19 M. Motamedifar, S. Mahdavifar and S. Shayesteh Farjami, J. Supercond. Nov. Magn. Online (2010).

20 M. Vojta, Rep. Prog. Phys. 66, 2069 (2003).

21 J. H. H. Perk, H. W. Capel, M. J. Zuilhof and Th. J. Siskens, Physica A 81, 319 (1975).

22 J. Abouie, S. Mahdavifar, Phy. Rev. B 78, 184437 (2008).

23 C. Lanczos, J. Res. Natl Bur. Stand. 45, 255 (1950).

24 G. Grosso, L. Martinelli, and G. Pastoriv Parravicini, Phys. Rev. B 51, 13033 (1995).

25 G. I. Japaridze, A. Langari, S. Mahdavifa, J. Phys.: Condens. MAtter 19, 076201 (2007).

26 S. Mahdavifar and A. Akbari, J. Phys. Soc. Jpn. 77, 024710 (2008). 